\title{
Cirrhotic Cardiomyopathy - a significant complication of Chronic Liver Disease Patients
}

\author{
S.Mahant ${ }^{1}$, N.Chandel ${ }^{2}$, Upasana ${ }^{3}$, P.D.Mahant ${ }^{4}$, T.Kumbhakar \\ ${ }^{1}$ Prof. Department of Medicine, PCMS \& RC, Bhopal, India \\ 2. Asst.Prof. Department of Radio diagnosis, PCMS \& RC, Bhopal,India \\ 3.Asst.Prof. Department of Pathology, PCMS \& RC, Bhopal,India \\ ${ }^{4}$ Prof. Department of Radio diagnosis, PCMS \& RC, Bhopal,India \\ ${ }^{5}$ Resident. Department of Medicine, PCMS \& RC, Bhopal, India
}

\begin{abstract}
Cirrhosis is very serious condition and associated with a range of cardiac abnormalities. However scanty information is available about the cardiac changes in cirrhotic patients in Indian setup, like increased resting cardiac output, blunted response to stress, left ventricular diastolic dysfunction and increased wall thickness of all cardiac chambers. These concomitant cardiac abnormalities in patients with cirrhosis have been termed as 'Cirrhotic Cardiomyopathy which may be a major cause of morbidity and mortality in patients with cirrhosis and liver transplant patients. With the advent of increased liver transplantation in India, this entity may have its impact on the transplantation success.
\end{abstract}

Key words: Cirrhotic echocardiography criteria, 6 minutes walking test

Methodology

Hundred patients with cirrhosis (alcoholic and non-alcoholic) were enrolled. Data collection was done by clinical history, examination, investigations. All subjects underwent Echocardiography study at rest and stress i.e. 6 minutes walking test.

Observation and Results-

Prevalence of cirrhotic cardiomyopathy in my study was $91.42 \%$.

Systolic dysfunction- At the time of stress, ejection fraction was $59.78 \pm 5.767$ in cirrhotic cardiomyopathy and $67 \pm 3.786$ in non- cirrhotic cardiomyopathy subjects. P value was 0.02 .

Diastolic dysfunction i.e. Deceleration time at rest in those having CC was $221.06 \pm 19.75$ and those not having CC was $193.66 \pm 20.87 \mathrm{~ms}$. E/A at stress in those having CC was $1.46 \pm 0.084$ and those not having was $1.30 \pm 0.399$.

\section{Introduction}

Chronic liver disease is the spectrum of a disease that involves a process of progressive destruction and regeneration of the liver parenchyma leading to fibrosis and cirrhosis. Clinical picture is dominated by signs of peripheral vasodilatation with palmer erythematic and reddish skin, raised and bounding pulse, and a low systemic blood pressure indicating a hyperdynamic circulation. The hyperdynamic syndrome comprises an increased heart rate, cardiac output, and plasma volume, and a reduced systemic vascular resistance and arterial blood pressure. Cirrhotic cardiomyopathy was initially thought to be of little clinical relevance. However, with the frequent use of invasive procedures like surgical portocaval shunts, transjugular intrahepatic port systemic shunt and liver transplantation, the adverse consequences of cardiac dysfunction became evident. Unfortunately there is scanty information on the status of cardiac abnormalities in Indian patients with cirrhosis ${ }^{1}$

\section{Aim and Objectives}

AIM- To study cardiac changes in patients of chronic liver disease

\section{Objectives-}

- To evaluate the extent of cardiac dysfunction by clinical and echocardiography assessment in chronic liver disease patients.

- To assess the prevalence of cirrhotic cardiomyopathy and correlation between cardiac dysfunction in patients of chronic liver disease to age.

$\bullet$

Material and Methods-

\section{Methodology}

This is a case series study, the study protocol was approved by the institutional ethics committee and written consent was obtained from each patient. Fifty consecutive patients with cirrhosis of liver presenting to 
Department of Medicine, People's College of Medical Sciences and Research Centre, Bhopal over three years were included in the study

Diagnosis of cirrhosis of the liver was made on clinical, biochemical, serological, and ultrasound imaging. . All patients were ambulatory and hemodynamically stable.

\section{Inclusion Criteria-}

1. Diagnosed cases of chronic liver disease irrespective of age and sex.

\section{Exclusion Criteria-}

1. $\mathrm{K} / \mathrm{C}$ of CAD, hypertension, RHD, NIDDM, cardiomyopathy

2. $\mathrm{K} / \mathrm{C}$ of hyperthyroidism, hypothyroidism

\section{Methodology-}

Hundred patients diagnosed as chronic liver disease clinically and supported by laboratory investigations irrespective of age, sex and aetiology were included in this study.

Liver function test was assessed by investigations including serum bilirubin, albumin, prothrombin time, ultrasonography, upper GI endoscopy. In addition s.creatinine, random blood suger, electrocardiogram was also tested. Then all the patients underwent cardiac evaluation and functional assessment by noninvasively, using transthorasic echocardiography performed at rest and after 6 minute stress. Detailed echocardiographic parameters including ejection fraction was calculated by using Modified Simpson rule, estimating E/A ratio, deceleration time, left ventricular wall thickness, septal wall thickness, and systolic and diastolic LV dimensions.

\section{Results-}

In the study hundred patients were included, age group ranging from 30 to 70 yrs, which included 82 males and 18 females constituting $82 \%$ and $18 \%$. Maximum age of the patient was 70 yrs and minimum was $30 \mathrm{yrs}$. Maximum patients come under the 50-59yrs age group (40\%).

Among the patients in the study, majority of the patients had alcoholic etiology (52\%). Non alcoholic etiologies were Hepatitis B $(42 \%)$ and Hepatitis C $(6 \%)$. None of the patients in the study had other etiologies like autoimmune, biliary causes. There were no patients of multiple etiologies.

The most common presentation was decrease appetite $(88 \%)$, abdominal distension $(88 \%)$, hematemesis and malena in $(54 \%)$ and common clinical signs were ascites $(94 \%)$, splenomegaly $(80 \%)$ edema $(68 \%)$, icterus $(48 \%)$ and pallor $(42 \%)$. Ascitis was minimal to gross was included in our study.

\section{Echocardiographic parameters of study population-}

Comparison between the echocardiographic parameters, of presence or absence of cirrhotic cardiomyopathy.

In our study 100 subjects were enrolled and out of it 84 have cirrhotic cardiomyopathy and 16 of them are not.

Echocardiographic parameters of study population have statistically significant difference of EF in stress (59.78 \pm 5.767$)$ in whom cirrhotic cardiomyopathy (CC) was present as compared those in whom cirrhotic cardiomyopathy was absent (67 \pm 3.786$)$. [P value is 0.02 ]

There was statistically significant difference of $\mathrm{E} / \mathrm{A}$ in stress $(1.46 \pm 0.084)$ in whom $\mathrm{CC}$ was present as compared those in which CC was absent $(1.30 \pm 0.399)$, $\mathrm{p}$ value is 0.03 .

There was also statistically significant difference of DT in rest $(221.061 \pm 19.75)$ in whom CC was present as compared those in which CC was absent (193.66 \pm 20.87$)$, $\mathrm{p}$ value are 0.0312 .

However, there were not statistically significant difference between other parameters like EF (rest), E/A (rest), DT (stress) and IVRT.

Table no.1- Echocardiographic parameters of study population

\begin{tabular}{|l|l|l|l|}
\hline $\begin{array}{l}\text { Echocardiographic } \\
\text { Parameters }\end{array}$ & $\begin{array}{l}\text { Cases-16(absence CC) } \\
\text { Mean } \pm \text { std deviation }\end{array}$ & $\begin{array}{l}\text { Cases }-84 \text { (Presence CC) } \\
\text { Mean } \pm \text { std deviation }\end{array}$ & P value \\
\hline EF (rest) & $58.67 \pm 3.786$ & $58.78 \pm 7.848$ & 0.980 \\
\hline EF (stress) & $67.67 \pm 3.786$ & $59.69 \pm 5.767$ & 0.026 \\
\hline E/A (rest) & $1.21 \pm 0.223$ & $1.28 \pm 0.460$ & 0.812 \\
\hline E/A (stress) & $1.30 \pm 0.399$ & $1.46 \pm 0.084$ & 0.0392 \\
\hline DT (rest) & $193.66 \pm 20.87$ & $221.06 \pm 19.75$ & 0.0312 \\
\hline DT (stress) & $201.33 \pm 57.63$ & $199.43 \pm 39.58$ & 0.939 \\
\hline IVRT (rest) & $99.00 \pm 29.20$ & $95.28 \pm 26.743$ & 0.820 \\
\hline IVRT (stress & $98.33 \pm 4.93$ & $98.75 \pm 35.71$ & 0.984 \\
\hline
\end{tabular}


Table no-2 Comparison between echocardiography parameters of alcoholic and non- alcoholic-

\begin{tabular}{|l|l|l|l|}
\hline $\begin{array}{c}|c| \\
\text { Echocardiographic }\end{array}$ & Alcoholic(n-52) & Non alcoholic(n-48) & P value \\
\hline EF & $57.10 \pm 7.905$ & $61.29 \pm 6.4$ & 0.1079 \\
\hline E/A & $1.39 \pm 0.47$ & $1.09 \pm 0.34$ & 0.047 \\
\hline DT & $189.2 \pm 44.12$ & $262.9 \pm 96.9$ & 0.004 \\
\hline IVRT & $92.5 \pm 30.01$ & $100.1 \pm 20.42$ & 0.3905 \\
\hline
\end{tabular}

There was not statistically significant difference between ejection fraction, E/A ratio, DT, and IVRT of alcoholic and non alcoholic patients

In our study we compared Child- Pugh Score with Cirrhotic cardiomyopathy. In A group there were 20 patients had cirrhotic cardiomyopathy out of 30, while in B group 28 subjects had cirrhotic cardiomyopathy out of 32 and in $\mathrm{C}$ group 36 had cirrhotic cardiomyopathy out of 38 . We analyzed that there is no relation between severity of liver disease and cirrhotic cardiomyopathy. ( $p$ value- 0.446 )

Table no.-3 Comparison between Child- Pugh Score and Cirrhotic cardiomyopathy-

\begin{tabular}{|l|l|l|l|}
\hline \multirow{2}{*}{ Child- Pugh score } & \multicolumn{2}{|l|}{ Cirrhotic cardiomyopathy } & \multirow{2}{*}{ Total } \\
\cline { 2 - 3 } & Present & Absent & \\
\hline A & 20 & 10 & 30 \\
\hline B & 28 & 4 & 32 \\
\hline C & 36 & 2 & 38 \\
\hline Total & 84 & 16 & 100 \\
\hline
\end{tabular}

Other echocardiographic findings in our study were presence of LVH in 4 subjects (4\%), mild pericardial effusion in 6 patients $(6 \%)$, mild mitral regurgitation in 2 patients $(2 \%)$ and aortic regurgitation in 2 patient $(2 \%)$. $\mathrm{P}$ value is insignificant.

\section{Discussion}

The present study is a case series study consisting of fifty patients with cirrhosis who were inpatients in the department of Medicine, People's College of Medical Sciences \& RC from 2010 to 2013. All patients were ambulant, thus eliminating the effect of cardiac deconditioning due to bed rest. The patients were evaluated for presence of cirrhotic cardiomyopathy.

In our study male patients outnumbered females ( $82 \%$ vs. $18 \%)$. This is due to the distribution of cirrhosis in between the genders as well as life style difference.

The mean age of the patients in our study was around 43 years and subjects in the age group 30-70 years were included. Age was compared with presence of cirrhotic cardiomyopathy and we found that there was no correlation between them. $\mathrm{p}$ value was insignificant.

Among the subjects in the studied the most common aetiology was alcoholic that contributes $(52 \%)$ remaining $48 \%$ was contributed by viral aetiology i.e. HBV (42\%) and $\mathrm{HCV}(6 \%)$

Majority of subjects presented with history of decrease appetite (88\%), abdominal distension (88\%) as chief complaint. Clinically ascites was found in (94\%) of our patients and oedema was detected in $(68 \%)$, due to activated RAAS, hypoproteinemia and ascites compressing the abdominal inferior venacava. Icterus was detected in patients $(48 \%)$ of the study population.

Cirrhotic patients of our study had liver dysfunction of early, intermediate \& late severity 15 patients (30\%) were in Child-Pugh class A, 16 patients (32\%) were in Child-Pugh class B, 19 Patients (38\%) were in class $\mathrm{C}$. In our study there was no significant correlation between the severity of hepatic dysfunction and cardiac changes, which is consistent with one published report from India ${ }^{1}$.

As in our study we included 50 cirrhotic subjects and out of this 42 had cirrhotic cardiomyopathy, so prevalence in our study was $84 \%$. But studies done earlier had prevalence of $25.4 \%{ }^{10}$. This difference in prevalence may be due to different tool of stress.

In our study 30 no. of subjects $(60 \%)$ had blunted response in ejection fraction after stress, 12 no. subjects $(24 \%)$ had decreased ejection fraction after stress and 8 of them $(16 \%)$ had increased ejection after stress. [p value is 0.02 ]

We found that there was statistically significant difference between E/A at stress ( $\mathrm{p}$ value -0.0392) and DT at rest ( $\mathrm{p}$ value -0.0312 ) in cirrhotic cardiomyopathy and non-cirrhotic cardiomyopathy subjects. And lastly there was no statistically significant difference IVRT at rest and IVRT at stress in both groups and $\mathrm{p}$ values were 0.820 and 0.984 respectively.

But we found that there was significant difference between E/A and DT of alcoholic and non- alcoholic subjects. [p values were $0.047,0.004$ respectively]. The cardiac changes in our patients seems to be due to cirrhosis rather alcohol. Moreover in cirrhotic cardiomyopathy systolic and diastolic dysfunction both should be present and in our cases both entities were present. 


\section{Conclusion}

1 .Most common age group of cirrhosis in my study is 50-59 yrs and there is no correlation between age and cirrhotic cardiomyopathy.

2. Majority of subjects in study population is male (88.57\%).

3. Most common etiology in our study group is alcoholic i.e. $60 \%$.

4. There is no correlation between cirrhotic cardiomyopathy and severity of liver dysfunction.

5. Prevalence of cirrhotic cardiomyopathy in my study was $91.42 \%$. 64 patients had Cirrhotic cardiomyopathy was found.

6. The significance of mild cardiac dysfunction in patient of cirrhosis such as ours will require longitudinal follow up studies to ascertain the true significance of our finding with respect to further early management of such patients.

7. Clinical management of cirrhotic patients with early cardiac dysfunction remains uncertain .But there is a scope of future studies to look into the role of newer pharmacological agent such as Endothelin or RAAS antagonist even before this patient develop severe symptomatic cardiac dysfunction.

Conflicts of interest - The authors who have taken part in this study declared that they do not have anything to disclose regarding funding or conflict of interest with respect to this manuscript.

\section{Reference}

[1]. Alexander J, Mishra P, Desai N et al. Cirrhotic cardiomyopathy- Indian Scenario J Gastroenterol Hepatol. March 2007; 22 (3) 3959.

[2]. Iwakiri Y, Groszmann RJ. The hyperdynamic circulation of chronic liver diseases: from the patient to the molecule.Hepatology. 2006;43:S121-S131.

[3]. Møller S, Henriksen JH. Cirrhotic cardiomyopathy: a pathophysiological review of circulatory dysfunction in liver disease.Heart. 2002;87:9-15.

[4]. Alqahtani SA, Fouad TR, Lee SS. Cirrhotic cardiomyopathy. Semin Liver Dis. 2008;28:59-69.

[5]. Zambruni A, Trevisani F, Caraceni P, Bernardi M. Cardiac electrophysiological abnormalities in patients with cirrhosis. J Hepatol. 2006;44:994-1002.

[6]. Møller S, Henriksen JH. Cardiovascular complications of cirrhosis. Gut. 2008;57:268-278.

[7]. Cazzaniga M, Salerno F, Pagnozzi G, Dionigi E, Visentin S, Cirello I, Meregaglia D, Nicolini A. Diastolic dysfunction is associated with poor survival in cirrhotic patients with transjugular intrahepatic portosystemic shunt. Gut in press. (online access Nov2006); doi:10.1136/gut.2006.102467. 